Revue internationale de l'économie sociale

Recma

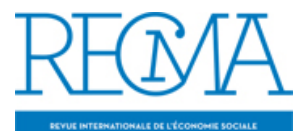

\title{
Les entreprises de l'économie sociale en question
}

\section{Jean-François Draperi}

Numéro 305, juillet 2007

URI : https://id.erudit.org/iderudit/1021505ar

DOI : https://doi.org/10.7202/1021505ar

Aller au sommaire du numéro

Éditeur(s)

Association Recma

ISSN

0035-2020 (imprimé)

2261-2599 (numérique)

Découvrir la revue

Citer ce document

Draperi, J.-F. (2007). Les entreprises de l'économie sociale en question. Revue internationale de l'économie sociale, (305), 4-5.

https://doi.org/10.7202/1021505ar d'utilisation que vous pouvez consulter en ligne.

https://apropos.erudit.org/fr/usagers/politique-dutilisation/ 


\section{LES ENTREPRISES DE L'ÉCONOMIE SOCIALE EN QUESTION}

L

es articles qui composent ce numéro 305 de la Recma portent sur l'entreprise d'économie sociale. Nous publions d'abord deux textes complémentaires sur la création et l'expansion des entreprises sociales en France. Thierry Sibieude s'intéresse à la création d'entreprise, vue sous l'angle des possibilités d'action des grandes entreprises d'économie sociale sur l'entrepreneuriat. Il souligne l'implication de certaines d'entre elles, qui réactualisent ainsi leur projet. Anne-Claire Pache et Géraldine Chalencon étudient l'expansion géographique des entreprises sociales, à partir d'une enquête menée auprès d'une vingtaine d'entre elles. Les modes d'expansion qu'elles définissent et leurs formes d'articulation revêtent un caractère heuristique remarquable.

Les deux textes qui suivent se penchent sur les coopératives latinoaméricaines: Maria Luiza Lins e Silva Pires explore le lien entre coopérative et territoire à partir de la présentation de coopératives agricoles au Brésil et en France. Juan Pablo Martí analyse le processus de récupération d'entreprise en Argentine et en Uruguay et porte son regard sur les relations avec les syndicats, relations dont nous savons la complexité historique.

Enfin, à partir de sa recherche-action québécoise, Daniel Côté poursuit l'étude du nouveau paradigme coopératif, en approfondissant le lien entre loyauté et coopérative et son impact sur la compétitivité de l'entreprise.

Chacun à sa façon, ces travaux mettent en évidence un risque relatif au maintien de l'identité. Dans le contexte économique de ce début de XXI ${ }^{e}$ siècle, que pèsent les spécificités des entreprises de l'économie sociale? Constituent-elles des handicaps ou des atouts? Inévitablement, la réponse est double: les spécificités sont plus ou moins marquées et elles sont à la fois des handicaps et des atouts. Il faut ajouter que cette réponse dépend non seulement des réalités de l'entreprise, mais aussi des questionnements des auteurs. En effet, si la grande diversité des situations objectives fait évidemment varier les réponses, on vérifie également que des auteurs différents peuvent soit mettre en évidence les processus de banalisation, soit, au contraire, mettre en avant les spécificités des mêmes entreprises, selon l'angle de leur analyse et le modèle théorique de référence. Or, nous vivons sans doute un tournant dans l'évolution des modèles de référence utilisés par les auteurs de la Recma. 
Jusque vers les années 1995, la Recma publiait des textes essentiellement rédigés par des spécialistes de l'économie sociale, chercheurs ou acteurs, peu nombreux, engagés, connaissant de près les mouvements et leur histoire. Au cours des dix années suivantes, nous avons accueilli un nombre toujours plus large de nouvelles signatures: fréquemment des jeunes auteurs qui étudiaient en profondeur un terrain appréhendé dans le cadre d'une thèse ou d'un mémoire. Ils sont malheureusement relativement peu nombreux à avoir poursuivi une carrière universitaire. Enfin, nous avons reçu au cours des deux dernières années un nombre croissant de textes d'universitaires plus avancés dans leur carrière, initialement non spécialistes de l'économie sociale, qui, suite à un événement déclencheur comme un appel d'offres de recherche, par exemple, choisissent de s'investir dans l'économie sociale. L'un des intérêts de ces travaux est de permettre une confrontation des entreprises de l'économie sociale aux modèles théoriques classiques, dont la validité a été vérifiée dans l'entreprise capitaliste. L'un des risques qu'ils comportent est de gommer les caractères spécifiques de l'économie sociale, caractères qui, par définition, n'apparaissent pas dans ces modèles théoriques: la double relation qui produit la double qualité ou la forme de solidarité que représentent les réserves impartageables, par exemple. Cette nouvelle diversité des analyses est une richesse. Mais il faut prendre garde au risque de ne plus prendre en considération les spécificités structurelles de l'économie sociale. Le contexte hyper-concurrentiel rend aujourd'hui plus primordiale la tâche de mieux faire connaître et partager la réalité des entreprises d'économie sociale. Ainsi que le propose Daniel Côté dans sa contribution à ce numéro, "alors qu'bistoriquement les coopératives étaient perçues comme une solution aux échecs de marché [...], il importe maintenant de regarder le mode d'organisation coopératif d'un point de vue proactif, c'est-à-dire de répondre à la question suivante: quels sont les avantages propres à la nature et à la structure de ce mode d'organisation en contexte concurrentiel? Cette approche nélimine aucunement la pertinence d'une analyse des conditions réactives. Il est simplement indispensable de réaliser la nécessité d'analyser également la pertinence au-delà de l'approche traditionnelle. "C'est à cette condition que sera maintenue la diversité théorique dont nous avons tous besoin. 